\title{
Scalp-recorded-EEG-based BFCN for Diagnosing AD and FTD Patients and Observing their Prognoses: Preliminary Results
}

\author{
Urata $\mathbf{R}^{1}$, Toh $\mathrm{T}^{2}$, Nakawatase $\mathrm{A}^{3}$, Yamazaki $\mathbf{T}^{1^{*}}$, Kuroiwa $\mathrm{Y}^{4}$, Baba $\mathrm{Y}^{5}$, Fujino $\mathrm{K}^{5}$ and Kurokawa $\mathrm{T}^{5}$
}

${ }^{1}$ Kyushu Institute of Technology, Fukuoka, Japan

${ }^{2}$ Nihon Unisys, Tokyo, Japan

${ }^{3}$ MOM Technology Co. Ltd., Fukuoka, Japan

${ }^{4}$ Medical Office, Ministry of Finance, Tokyo, Japan

${ }^{5}$ University Hospital, Mizonokuchi Teikyo University School of Medicine, Kanagawa, Japan

\begin{abstract}
In order to develop an easier and more inexpensive tool than using MEG and fMRI for diagnosing neurological diseases such as AD and FTD and checking their prognoses in future, we constructed scalp-recorded-EEGbased brain functional connectivity networks (BFCNs), and preliminarily compared Alzheimer's disease (AD) and frontotemporal dementia (FTD) patients, their prognoses and control subjects by the BFCNs. The present comparison among AD, FTD and older controls roughly supported the previous findings for synchronization likelihood values, unweighted graphs, clustering coefficient and characteristic path length. However, there were reverse differences in small-worldness. For AD and FTD, there were several electrode positions with higher betweenness centrality than the older controls. It might be suggested that we should investigate the betweenness centrality in more details.
\end{abstract}

Keywords: Alzheimer's disease; Frontotemporal dementia; Scalprecorded-EEG-based brain functional connectivity networks; Neurophysiological data

\section{Introduction}

Recently, there have been increasing applications of brain functional connectivity networks (BFCNs) [1] to patients and healthy subjects with neurophysiological data containing EEGs [2,3], MEG [4] and fMRI [5]. The psychiatric disorders are wide-ranging such as mild cognitive impairment (MCI) [5], post-traumatic stress disorder (PTSD) [2], frontotemporal dementia (FTD) [6] and Alzheimer's disease (AD) [3].

There had been already some functional connectivity measures. The classical coherency is not suitable to characterize non-stationary data with rapidly changing interdependencies. The phase synchronization [7] is valid only when the time series are approximately oscillatory. The synchronization likelihood (SL) [8] is a measure of the generalized synchronization between any two dynamical systems. This measure is closely related to the concept of generalized mutual information, and can also be computed in a time-dependent way, leading us to the analysis of non-stationary data.

Recently, among the parameters characterizing the BFCNs, the betweenness centrality $[2,3,5]$ has been often used in addition to clustering coefficients and characteristic path length, both of which are associated with the small-worldness.

In this preliminary study, using the scalp-recorded EEGs, BFCNs for the AD and FTD patients, their prognoses and control subjects with "older" and "younger" are constructed by the SL and various measures and parameters of the BFCNs are investigated, which will yield tools for diagnosing $\mathrm{AD}$ and FTD patients and checking their prognoses in future.

\section{Materials and Methods}

Figure 1 shows the flow from EEG recordings to BFCN construction in this study.

\section{Subjects}

Three female AD and one FTD patients and nine controls of 60-71 years ("older") and 21-24 ones (“younger") participated in this study after giving written, informed consent, which was approved by the ethics committees for Human Subject Researches, Faculty of Computer Science and Systems Engineering, Kyusyu Institute of Technology and for University Hospital, Mizonokuchi Teikyo University School of Medicine. The subject characteristics are summarized in Table 1. This study involved patients and older controls referred to the University Hospital. These subjects were studied according to a clinical protocol which involved history taking, physical and neurological examination, blood tests, neuropsychological examination, magnetic resonance imaging of the brain, and a quantitative EEG. The final diagnosis was based on a consensus meeting where all the available clinical data and the results of the ancillary investigations were considered. Four females of the "older" controls had symptoms of labyrinthine dizziness, body stagger, hand stiffness and TGA (transient global amnesia), respectively. For one of the three AD patients, EEG measurement was carried out also about one year after the previous one. At this time, EEGs were measured for the FTD patient and the "older" controls.

\section{EEG analysis}

EEG data acquisition: EEG data acquisition was performed through a Nihon Kohden EEG-1224 and a DIGITEX LAB Polymate AP1132 for the patients and the healthy controls, respectively. The device was equipped with $16 \mathrm{Ag} / \mathrm{AgCl}$ electrodes (a Nihon Kohden H503A) and active ones (a DIGITEX LAB AP-C100-0155), respectively. Both of the electrode positions were Fp1, Fp2, F3, F4, C3, C4, P3, P4, O1, O2, F7,

*Corresponding author: Yamazaki T, Kyushu Institute of Technology, Fukuoka Japan, Tel: 81-948-29-7818; Fax: 81-948-29-7801; E-mail: t-ymzk@bio.kyutech.ac.jp

Received February 02, 2016; Accepted February 25, 2016; Published February 29, 2016

Citation: Urata R, Toh T, Nakawatase A, Yamazaki T, Kuroiwa Y, et al. (2016) Scalp-recorded-EEG-based BFCN for Diagnosing AD and FTD Patients and Observing their Prognoses: Preliminary Results. J Comput Sci Syst Biol 9: 038044. doi:10.4172/jcsb.1000219

Copyright: (c) 2016 Urata R, et al. This is an open-access article distributed under the terms of the Creative Commons Attribution License, which permits unrestricted use, distribution, and reproduction in any medium, provided the original author and source are credited. 

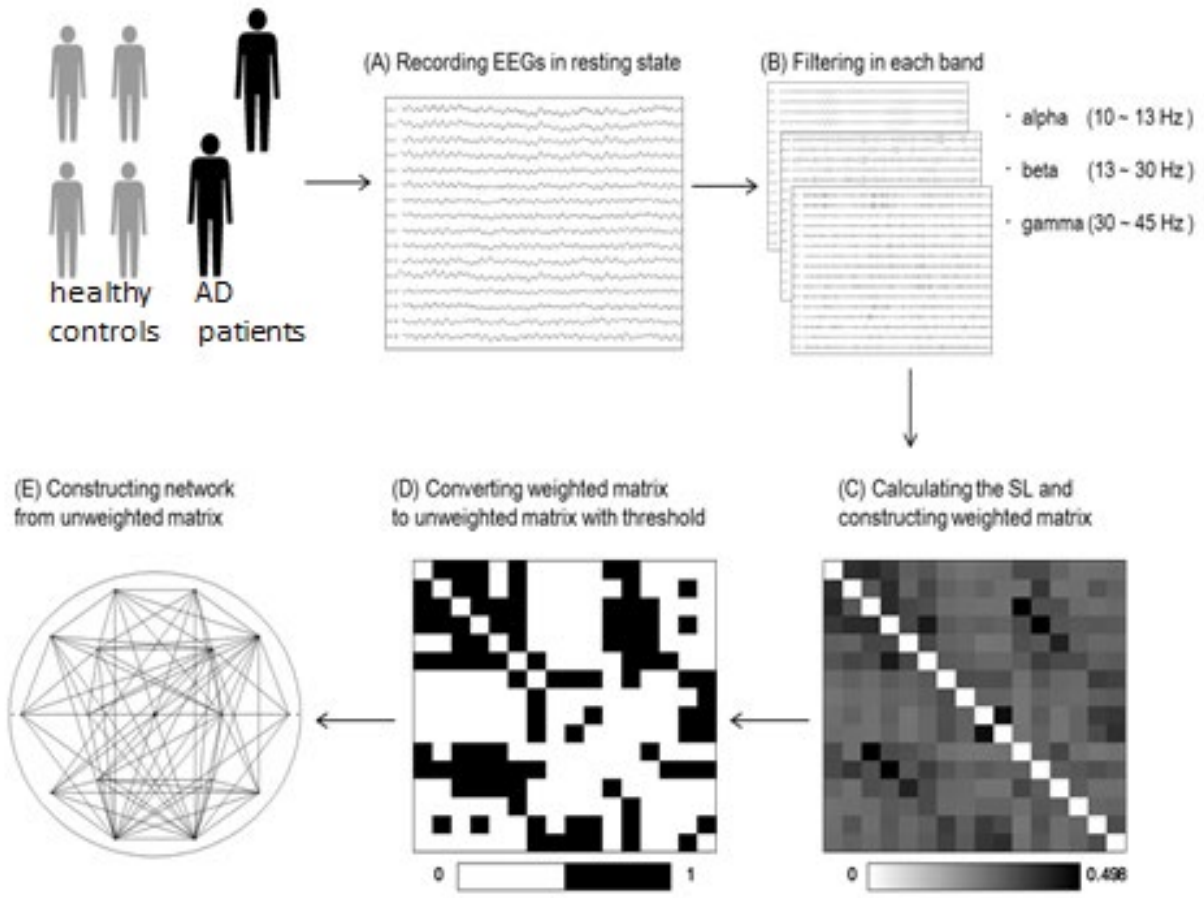

Figure 1: Flow from EEG recordings to BFCN construction.

\begin{tabular}{|l|c|c|c|}
\hline Subject Characteristics & AD & FTD & \multicolumn{2}{|c|}{ Controls } \\
\hline & & & Older \\
\hline Age $^{*}$ & $88(86-90)$ & 82 & $66.4(60-71)$ \\
\hline$M: F^{* *}$ & $0: 3$ & $0: 1$ & $22.8(21-24)$ \\
\hline
\end{tabular}

: mean (range) in years

$\mathrm{M}=$ male, $\mathrm{F}=$ Female

Table 1: The Summary of subject characteristics.

F8, T3, T4, T5, and T6 on the basis of the International 10-20 System. Electrode impedance was below $5 \mathrm{k} \Omega$. The filter setting was: High pass filter $=120 \mathrm{~Hz}$. Sample frequency was $500 \mathrm{~Hz}$ and A-D precision 32 bit. All the subjects were instructed to lie on their sides in the resting state with their closed eyes, for $5 \mathrm{~min}$ at least. For further offline postprocessing, from 1 minute after the EEG measurement onset, we set a window consisting of 500 sampling points every 1 second, selected the EEG corresponding to 100 windows, and the data was band-pass filtered for the commonly used frequency bands: lower alpha $(8-10 \mathrm{~Hz})$, upper alpha $(10-13 \mathrm{~Hz})$, beta $(13-30 \mathrm{~Hz})$ and gamma $(30-45 \mathrm{~Hz})$. All further analyses were performed for these frequency bands separately. In the following, averages of the 100 windows were described for all values and measures.

Synchronization analysis: The synchronization analysis involved each sequential 500-sampling-point. Correlations between all pairwise combinations of EEG channels were computed with the SL. Mathematical details for the SL can be found in Ref. [8] and Ref. [9]. The SL is a general measure of the correlation or synchronization between two time series that is sensitive to linear as well as non-linear interdependencies. The basic principle of the SL is to divide each time series into a series of "pattern" (roughly, brief pieces of time series containing a few cycles of the dominant frequency) and to search for a recurrence of these patterns. The SL is then the probability that the pattern recurrence in time series $X$ coincides in time with the pattern recurrence in time series Y. The end result of computing the SL for all pair-wise combinations of channels is a square matrix, where each entry contains the resulting SL value of the sensor pair. This matrix is called the weighted (connection strengths or weights are included) adjacency or connectivity matrix A. Note that any connectivity measure could be used for this purpose. Since all connections in our network are bidirectional, the adjacency matrix is symmetrical along its diagonal axis (Figure 1).

\section{Network analysis}

Synchronization matrix thresholding: The weighted matrix was then passed through a threshold to be transformed into binary, resulting in undirected graphs. To avoid the influence of methodological limitations posed by BFCNs originally depending on degree [10] and sparsity [11], the threshold should be adaptively selected. In this study, assuming that the BFCNs of all the subjects have the same number of edges, the average degree $(K)$ of all nodes was set, and then the threshold was determined so that each $K$ was obtained. So, both global and local properties of the BFCNs (clustering coefficient, characteristic path length, small-worldness, betweenness centrality) were quantified 
Citation: Urata R, Toh T, Nakawatase A, Yamazaki T, Kuroiwa Y, et al. (2016) Scalp-recorded-EEG-based BFCN for Diagnosing AD and FTD Patients and Observing their Prognoses: Preliminary Results. J Comput Sci Syst Biol 9: 038-044. doi:10.4172/jcsb.1000219

for each subject. The following subsection provides a brief description of these BFCN measures.

Description of network parameters: Basic elements in the graph theory are illustrated shown in Figure 2. Six circles are called nodes, lines connecting any two nodes edges and distances between any two nodes in terms of the summation of edges connecting the nodes paths. The degree $(k)$ of a node is the number of connections to the node. The mean degree $(K)$ is obtained by dividing the total number of edges by the total number of nodes in a network. For the undirected and unweighted graph structure shown in Figure 2, the degree of the node "parietal association cortex" is 4 and the mean degree is 1.5. Moreover, the network parameters characterizing the graph are described in the following.
Clustering coefficient (C): This measure can quantify the local connectivity in individual nodes or the network. For a node, the neighbors are other nodes which are connected to one node. The clustering coefficient is determined by the ratio between the number of edges by which the neighbors are actually connected and the maximum number of edges that can be connected to each other in the neighbors.

Characteristic path length (L): This measure can quantify the global connectivity of the network is able to build an optimal path. The characteristic path length is the shortest path between the nodes.

Small-worldness: When whether the above measures in the graph theory (the clustering coefficient and characteristic path length) are

\section{healthy subject 1}

\section{healthy subject 2}
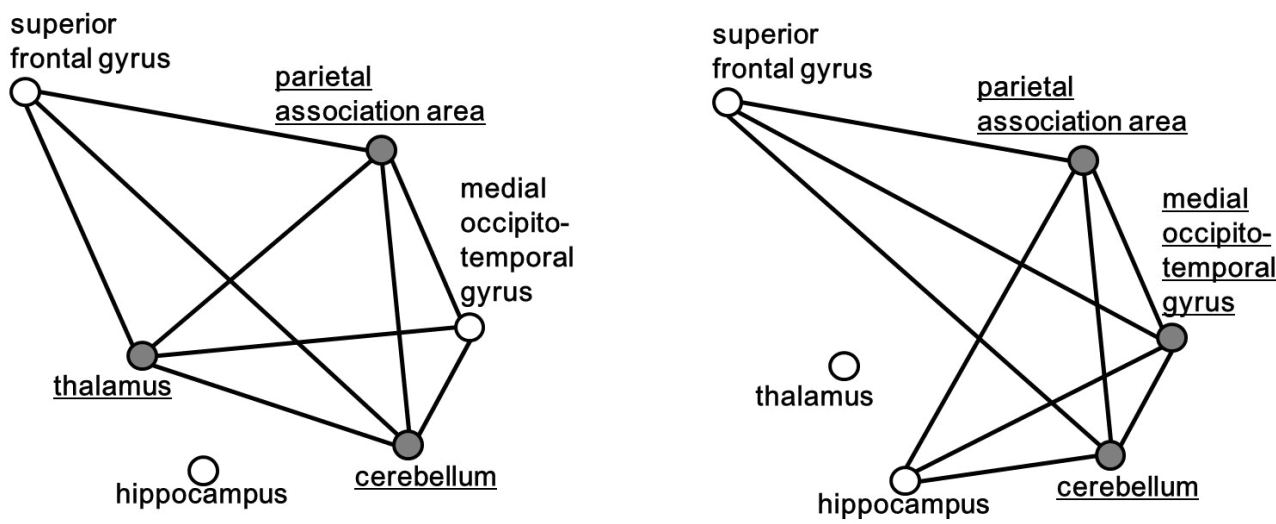

Figure 2: ECDL-based BFCN for healthy controls.
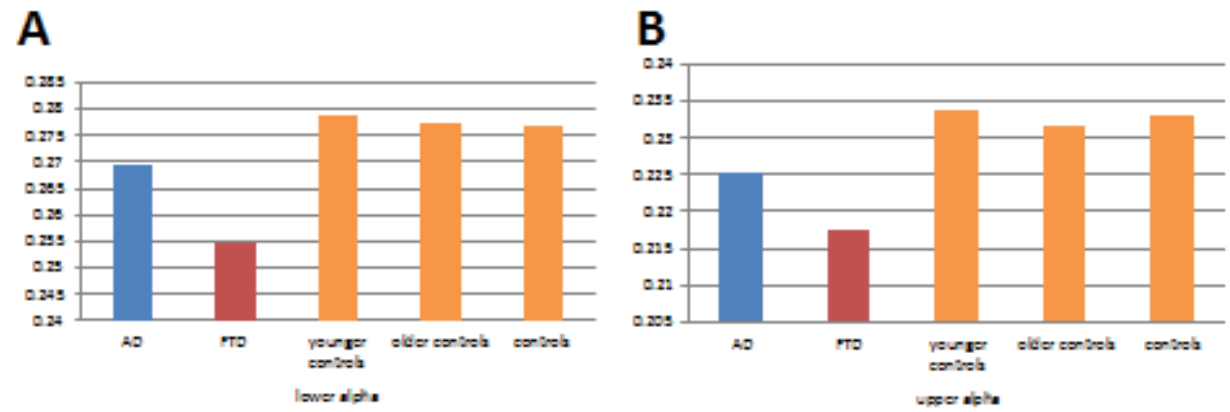

\section{C}

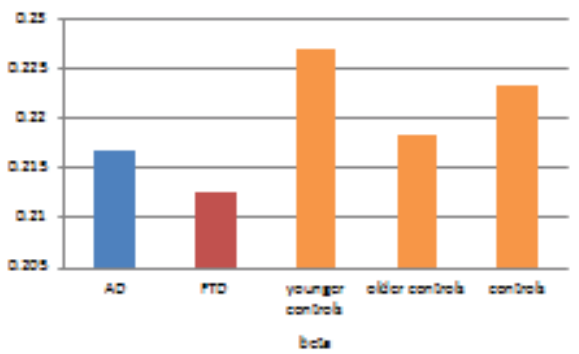

D

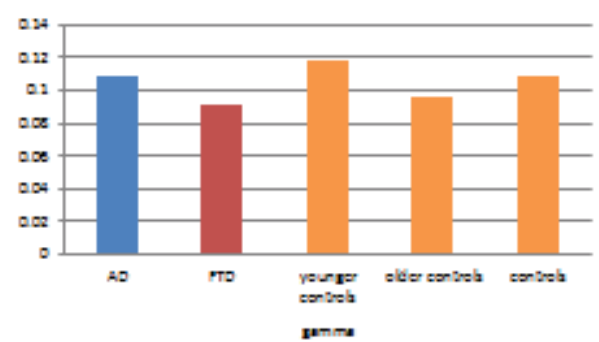

Figure 3: Mean SL values of each frequency band (A: lower $\alpha$; B: upper $\alpha$; C: $\beta$; D: $\gamma$ ) for AD, FTD, younger controls, older controls and all the controls. 


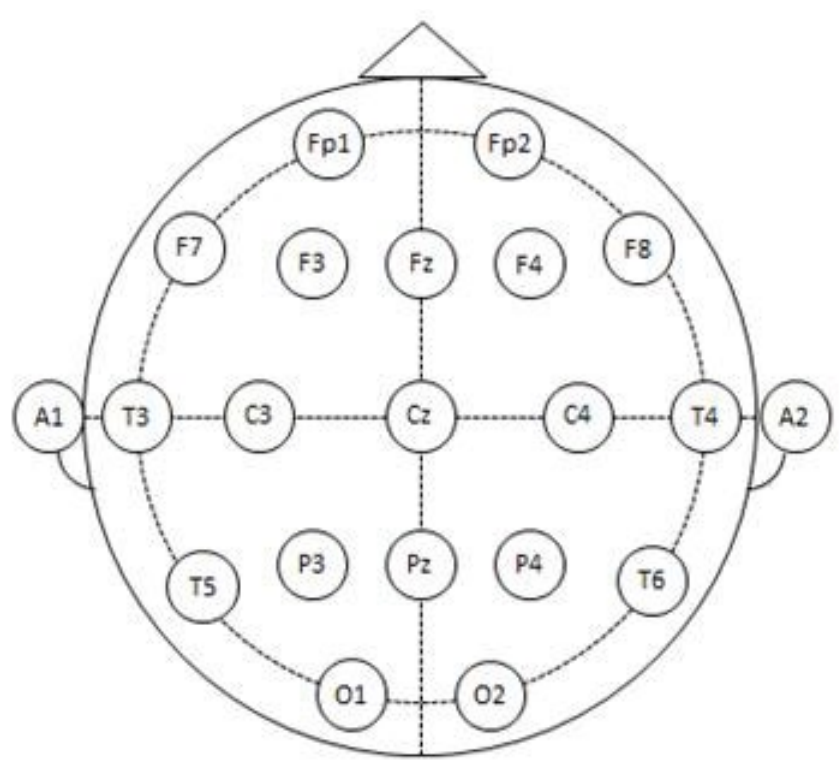

Figure 4: Schematic image of the head seen from above, with the positions of the electrodes indicated by circles and numbered the 10-20 electrode placement system.

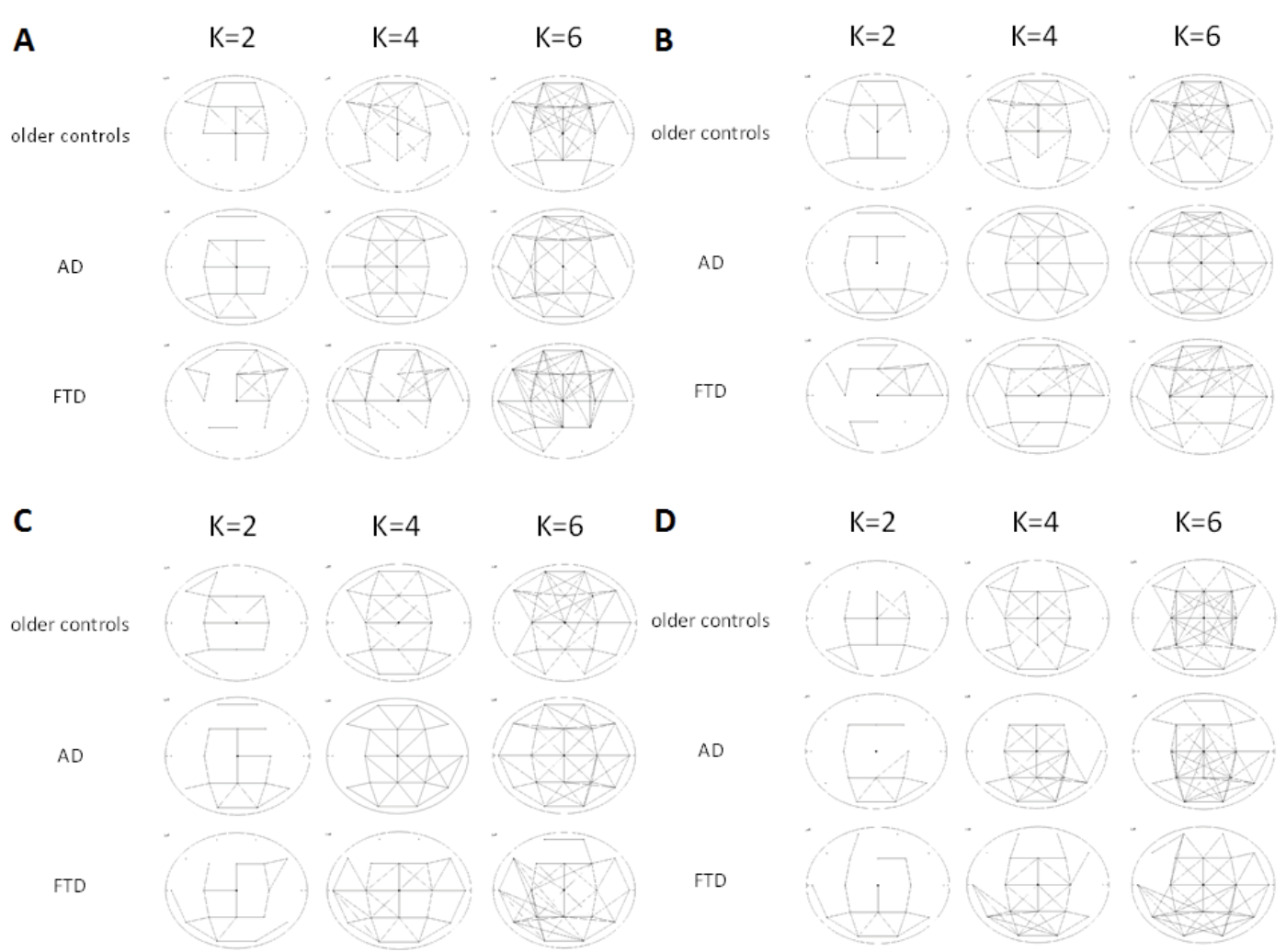

Figure 5: Unweighted graphs of four frequency bands (A: lower alpha; B: upper gamma; C: beta; D: gamma) for AD, FTD and older controls and different fixed average degrees $(K)$. See Figure 4 for node positions.

significant or not is examined, a network with randomly rewiring edges is constructed by fixed nodes and edges. This network is called the random network, and it is necessary to normalize the measures by using the random network [12].
When the clustering coefficient and the characteristic path length in the random network constructed by "Edge Switching Algorithm (ESA)" [12] are respectively represented by $C_{r}$ and $L_{r}$, and their normalized measures are given by $C_{n}=C_{p} / C_{r}$ and $L_{n}=L_{p} / L_{r}$, respectively, 
Citation: Urata R, Toh T, Nakawatase A, Yamazaki T, Kuroiwa Y, et al. (2016) Scalp-recorded-EEG-based BFCN for Diagnosing AD and FTD Patients and Observing their Prognoses: Preliminary Results. J Comput Sci Syst Biol 9: 038-044. doi:10.4172/jcsb.1000219

where $C_{p}$ and $L_{p}$ are the clustering coefficient and characteristic path length. Then, the small-worldness $(S)$, one of the measures for smallworld property, is given by $S=C_{n} / L_{n}$.

Betweenness centrality: The centrality is a measure that indicates how important nodes in a network are. Definitions of "important" depend on the type of centrality. In this study, the importance of the node is assumed to be how much the removal of the nodes in the constructed network influences the efficient connection. In the betweenness centrality, when the node passes the shortest path, the node has high centrality. Therefore, the betweenness centrality is used also to compare with the previous studies under the same conditions. The betweenness centrality $B_{i}$ of a node $i$ was defined as the number of shorter paths between any pair of nodes that run through node $i$ [13].

\section{Results}

\section{Averaged SL}

Figure 3 shows mean SL values of each frequency band for $\mathrm{AD}$, FTD, younger controls, older controls and all the controls. In AD and FTD, the mean SL decreased compared to the older controls except for the gamma band (Figure 4).

\section{Unweighted graphs of the BFCNs}

Figure 5 shows unweighted graphs of four frequency bands (A: lower alpha; B: upper gamma; C: beta; D: gamma) for AD, FTD and older controls and different fixed average degrees $(K)$. For all the frequency bands, AD and FTD graphs have fewer connections, especially in the frontal nodes, than the older controls.

\section{Clustering coefficient, characteristic path length and small- worldness}

Figure 6 shows clustering coefficient (A), characteristic path length (B) and small-worldness (C) of four frequency bands in each $K$ for AD and older controls, where "ave" is the average of all the Ks. Roughly, in $\mathrm{AD}$, the clustering coefficient increased compared to the older controls, and the characteristic path length increased or not changed. On the other hand, in $\mathrm{AD}$, the small-worldness increased compared to the older controls.

\section{Betweenness centrality}

Figure 7 shows betweenness centrality of three frequency bands at each electrode position for $\mathrm{AD}$ and older controls, where "ave" is the average of all the electrode positions. Commonly to the lower and upper alpha and the beta, $\mathrm{AD}$ had higher betweenness centrality at the midline (Fz, Cz), the bilateral temporal (T3, T4, T5, T6) and occipital $(\mathrm{O} 1, \mathrm{O} 2)$ than the older controls. In addition to the above electrodes, FTD had higher one at F7 and F8 (Figure 8).

\section{Discussion}

The present comparison among AD, FTD and older controls roughly supported the previous findings for synchronization likelihood values, unweighted graphs, clustering coefficient and characteristic path length.

However, in this study, there were reverse differences in smallworldness. Stam et al. [14], Supekar et al. [10] and De Haan et al. [15] found that the $\mathrm{AD}$ patients had lower small-worldness than the healthy controls, and demonstrated that $\mathrm{AD}$ is characterized by loss of small-
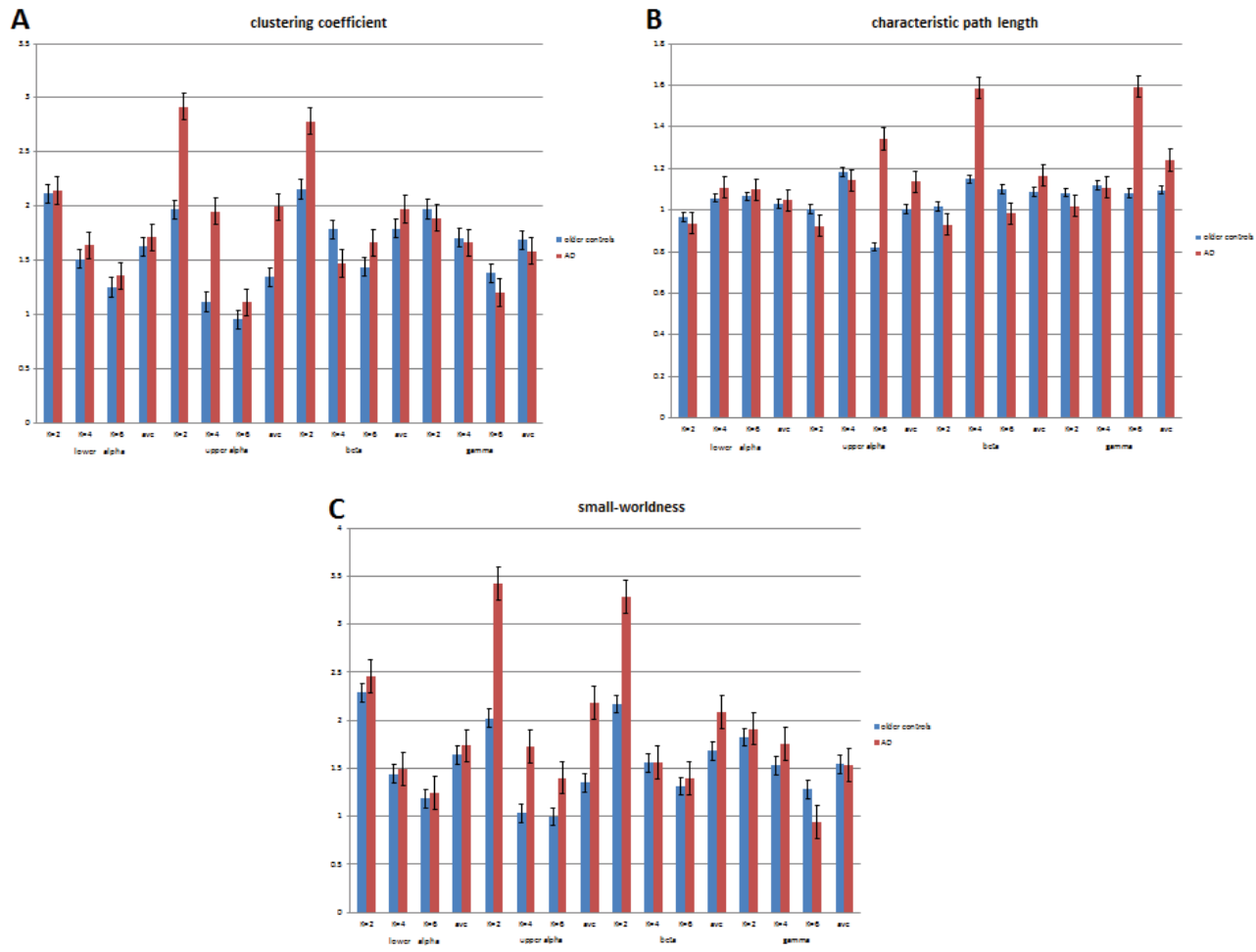

Figure 6: Clustering coefficient $(A)$, characteristic path length $(B)$ and small-worldness $(C)$ of four frequency bands (lower, upper alpha, beta and gamma) in each $K$ for $\mathrm{AD}$ and older controls, where "ave" is the average of all the $K s$. 

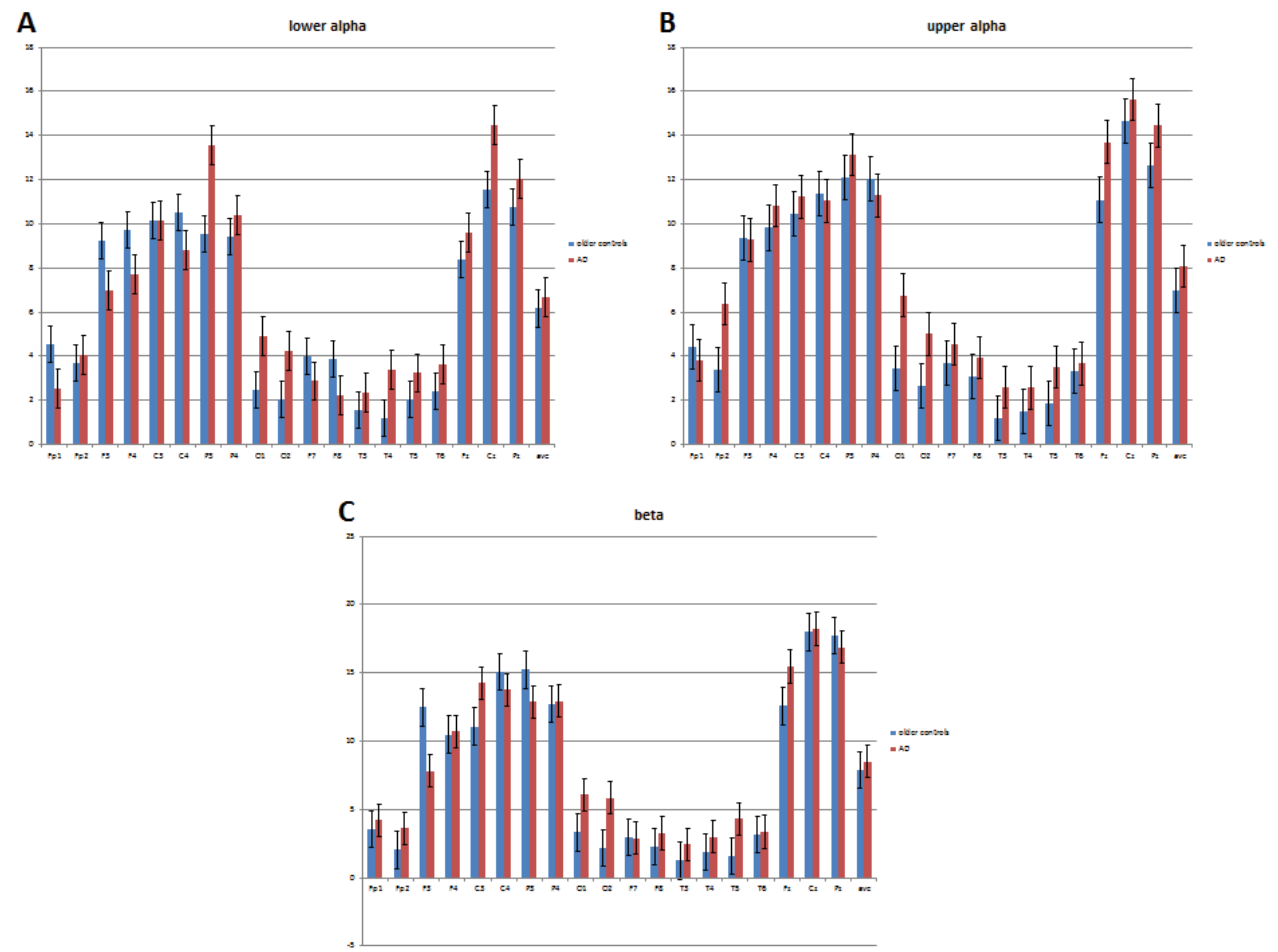

Figure 7: Betweenness centrality of three frequency bands (lower (A), upper (B) alpha and beta (C)) at each electrode position for AD and older controls, where "ave" is the average of all the electrode positions.

A

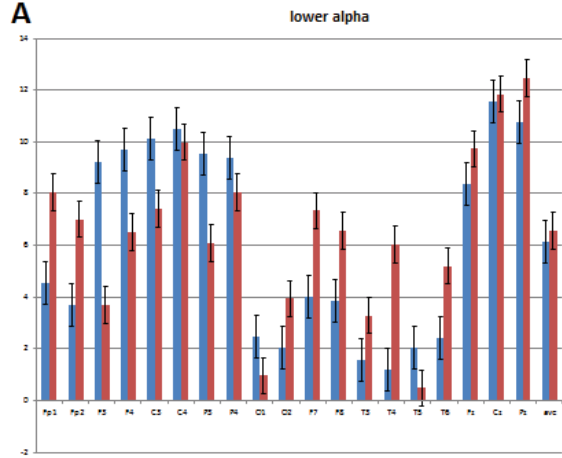

C
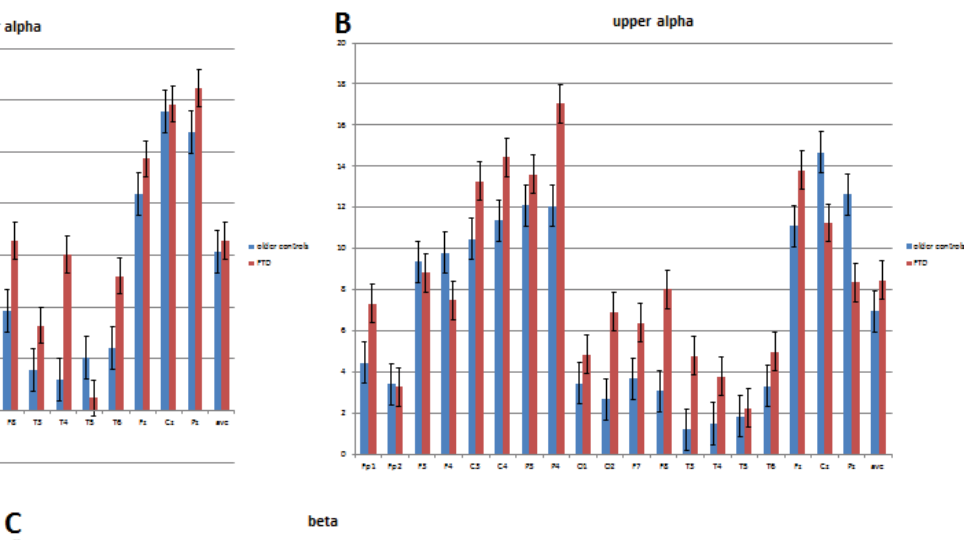

beta

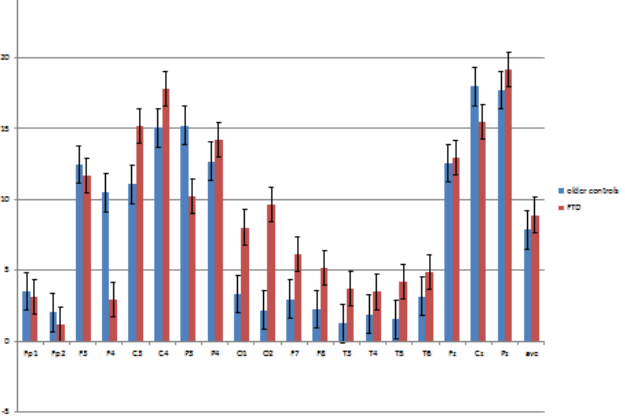

Figure 8: Betweenness centrality of three frequency bands (lower (A), upper (B) alpha and beta (C)) at each electrode position for FTD and older controls, where "ave" is the average of all the electrode positions. 
Citation: Urata R, Toh T, Nakawatase A, Yamazaki T, Kuroiwa Y, et al. (2016) Scalp-recorded-EEG-based BFCN for Diagnosing AD and FTD Patients and Observing their Prognoses: Preliminary Results. J Comput Sci Syst Biol 9: 038-044. doi:10.4172/jcsb.1000219

worldness. On the other hand, subsequent fMRI studies $[5,11,16,17]$ have appealed that $\mathrm{AD}$ is characterized by higher clustering coefficient and longer characteristic path length than healthy controls, not lower small-worlness. Although these discrepancies may be due to methological differences (EEG vs. fMRI) [1], our results supported the above fMRI ones, similarly to Ref. [14]. The clustering coefficient, the characteristic path length and also the small-worldness depend on thresholding [14], degree [10] and sparsity [11]. A new measure (cf. [18] for the small-worldness) might be needed which would have few effects of these parameters.

Differences in betweenness centrality between $\mathrm{AD}$ patients and healthy subjects using fMRI was firstly revealed by He et al. [11]. Frontal, central, temporal to occipital regions over the whole brain showed high betweenness centrality in the BFCNs of the AD patients, which was supported by Ref. [5,16]. We also obtained higher betweenness centrality than the older controls at several electrode positions such as the frontal, temporal and occipital ones. These results may indicate abnormal cerebral structures accompanied by atrophy of the gray matter in AD patients [19]. In the previous EEG studies, however, there was no significant difference in betweenness centrality between PTSD [2], aMCI and MD [3], and healthy subjects. It is suggested that the betweenness centrality should be investigated in more details.

Andreou et al. [20] revealed the BFCN of increased resting-state gamma-band connectivity in patients with schizophrenia compared to healthy controls, using EEG, MEG and fMRI. The connectivity between any two nodes was quantified by power envelope correlation [21]. The intracortical sources of brain electrical activity as the nodes were localized using exact low-resolution electromagnetic tomography (eLORETA) [22]. By the similar approach to Andreou et al. [20], we obtained the BFCNs of resting-state in healthy subjects using SL. In Figure 2, the nodes, indicated by open circles, which correspond to the brain regions where equivalent current dipoles (ECDs) were located by ECD source localization (ECDL) using the 19-ch scalp-recorded EEGs, where grey circles represent the nodes having the maximum betweenness centrality. From multi-channel EEGs, the brain regions could be specified by the ECDL. The ECDL is a method for localizing the neural generators as physical current dipole sources using multichannel EEGs or MEGs. Mathematical details for the ECDL can be found in Ref. [23]. Here, independent component analysis (ICA) [24] was applied to the multi-channel EEGs, and then ICs after deflation were analyzed by the ECDL. The "parietal association area" node in Figure 2 contains the angular and supramarginal gyri and the superior parietal lobule where dipoles were located by the ECDL. Therefore, Figure 2 partly indicates the same results as those in Liu et al. [5] for the healthy subjects.

SL values, unweight graphs, clustering coefficient, characteristic path length and betweenness centrality of BFCNs could be promising for the diagnostic and prognosis-observable tool using scalp-recorded EEGs.

\section{Acknowledgements}

The authors thank Dr. B. W. Van Dijk, Magnetoencephalography Center, VU University Medical Center, Amsterdam, who consulted the convenience of the academic free software for calculating the SL.

\section{References}

1. Sporns O (2010) Networks of the Brain. Cambridge, Massachusetts: MIT Press, UK.

2. Lee SH, Yoon S, Kim JI, Jin SH, Chung CK (2014) Functional connectivity of resting state EEG and symptom severity in patients with post-traumatic stress disorder. Progress in Neuro-Psychopharmacology \& Biological Psychiatry 51: $51-57$
3. Frantzidis CA, Vivas AB, Tsolaki A, Klados MA, Tsolaki M, et al. (2014) Functional disorganization of small-world brain networks in mild Alzheimer's disease and amnestic mild cognitive impairment: An EEG study using relative wavelet entropy. Front Aging Neurosc 6: 224.

4. De Haan W, Van der Flier WM, Wang $H$, Van Mieghem PFA, Scheltens $P$, et al. (2012) Disruption of functional brain networks in Alzheimer's disease: What can we learn from graph spectral analysis of resting-state magnetoencephalography Brain Connect 2: 45-55.

5. Liu Z, Zhang Y, Yan H, Bai L, Dai R, et al. (2012) Altered topological patterns of brain networks in mild cognitive impairment and Alzheimer's disease: a restingstate fMRI study. Psychiatry Res 202: 118-125

6. Hodges JR, Patterson K (2007) Semantic dementia: a unique clinicopathological syndrome. Lancet Neurol 6: 1004-1014.

7. Mheich A, Hassan M, Khalil M, Berrou C, Wendling F (2015) A new algorithm for spatiotemporal analysis of brain functional connectivity. J Neurosci Methods 242: 77-81.

8. Stam CJ, Van Dijk BW (2002) Synchronization likelihood: an unbiased measure of generalized synchronization in multivariate data sets. Physica 163: 236-51.

9. Montez T, Linkenkaer-Hansen K, van Dijk BW, Stam CJ (2006) Synchronization likelihood with explicit time-frequency priors. Neuroimage 33: 1117-1125.

10. Supekar K, Menon V, Rubin D, Musen M, Greicius MD (2008) Network analysis of intrinsic functional brain connectivity in Alzheimer's disease. PLoS Comput Biol 4: e1000100.

11. He Y, Chen Z, Evans A (2008) Structural insights into aberrant topological patterns of large-scale cortical networks in Alzheimer's disease. J Neurosci 28 : 4756-4766.

12. Milo R, Shen-Orr S, Itzkovitz S, Kashtan N, Chklovskii D, et al. (2002) Network motifs: simple building blocks of complex networks. Science 298: 824-827.

13. Freeman LC (1977) A set of measures of centrality based on betweenness. Sociometry 40: 35-41.

14. Stam CJ, Jones BF, Nolte G, Breakspear M, Scheltens P (2007) Small-world networks and functional connectivity in Alzheimer's disease. Cereb Cortex 17: 92-99.

15. de Haan W, Pijnenburg YA, Strijers RL, van der Made Y, van der Flier WM, et al. (2009) Functional neural network analysis in frontotemporal dementia and Alzheimer's disease using EEG and graph theory. BMC Neurosci 10: 101.

16. Yao Z, Zhang Y, Lin L, Zhou Y, Xu C, et al. (2010) Abnormal cortical networks in mild cognitive impairment and Alzheimer's disease. PLoS Comput Biol 6 : e1001006.

17. Zhao X, Liu Y, Wang X, Liu B, Xi Q, et al. (2012) Disrupted small-world brain networks in moderate Alzheimer's disease: a resting-state FMRI study. PLoS One 7: e33540.

18. Muldoon SF, Bridgeford EW, Bassett DS (2015) Small-world propensity in weighted, real-world networks. Neuroscience S1: 1-13.

19. Rohrer JD (2012) Structural brain imaging in frontotemporal dementia. Biochim Biophys Acta 1822: 325-332.

20. Andreou C, Nolte G, Leicht G, Polomac N, Hanganu-Opatz IL, et al. (2015) Increased Resting-State Gamma-Band Connectivity in First-Episode Schizophrenia. Schizophr Bull 41: 930-939.

21. Hipp JF, Engel AK, Siegel M (2011) Oscillatory synchronization in large-scale cortical networks predicts perception. Neuron 69: 387-396.

22. Pascual-Marqui RD, Lehmann D, Koukkou M, Kochi K, Anderer P, et al. (2011) Assessing interactions in the brain with exact low-resolution electromagnetic tomography. Philos Trans A Math Phys Eng Sci 369: 3768-3784.

23. Yamazaki T, Tanaka K, Shibata T, Yamaguchi H, Ouda M, et al. (2014) Categorical-data-based $\mathrm{BCl}$ with motor imagery equivalent current dipole source localization. J Rehabilitation Robotics 2: 1-12.

24. Hyvärinen A, Oja E (1997) A fast fixed-point algorithm for independent component analysis. Neural Comput 9: 1483-1492. 